\title{
Thermal conductance of single-molecule junctions
}

Longji Cui ${ }^{1,6}$, Sunghoon Hur ${ }^{1}$, Zico Alaia Akbar ${ }^{2}$, Jan C. Klöckner ${ }^{3,4}$, Wonho Jeong ${ }^{1}$, Fabian Pauly ${ }^{3,4 *}$, Sung-Yeon Jang ${ }^{2,7 *}$, Pramod Reddy ${ }^{1,5 *} \&$ Edgar Meyhofer ${ }^{1 *}$

Single-molecule junctions have been extensively used to probe properties as diverse as electrical conduction ${ }^{1-3}$, light emission ${ }^{4}$, thermoelectric energy conversion ${ }^{5,6}$, quantum interference ${ }^{7,8}$, heat dissipation ${ }^{9,10}$ and electronic noise ${ }^{11}$ at atomic and molecular scales. However, a key quantity of current interest-the thermal conductance of single-molecule junctions - has not yet been directly experimentally determined, owing to the challenge of detecting minute heat currents at the picowatt level. Here we show that picowatt-resolution scanning probes previously developed to study the thermal conductance of single-metal-atom junctions ${ }^{12}$, when used in conjunction with a time-averaging measurement scheme to increase the signal-to-noise ratio, also allow quantification of the much lower thermal conductance of single-molecule junctions. Our experiments on prototypical Au-alkanedithiol-Au junctions containing two to ten carbon atoms confirm that thermal conductance is to a first approximation independent of molecular length, consistent with detailed ab initio simulations. We anticipate that our approach will enable systematic exploration of thermal transport in many other one-dimensional systems, such as short molecules and polymer chains, for which computational predictions of thermal conductance ${ }^{13-16}$ have remained experimentally inaccessible.

Studies of charge and heat transport in molecules are of great fundamental interest, and are of critical importance for the development of a variety of technologies, including molecular electronics ${ }^{17}$, thermally conductive polymers ${ }^{18}$ and thermoelectric energy-conversion devices ${ }^{19}$. Given this overall importance and the daunting experimental challenges, a number of initial studies explored charge transport in ensembles of molecules ${ }^{20,21}$. Although such measurements provided important insights, researchers gradually began to realize that it was

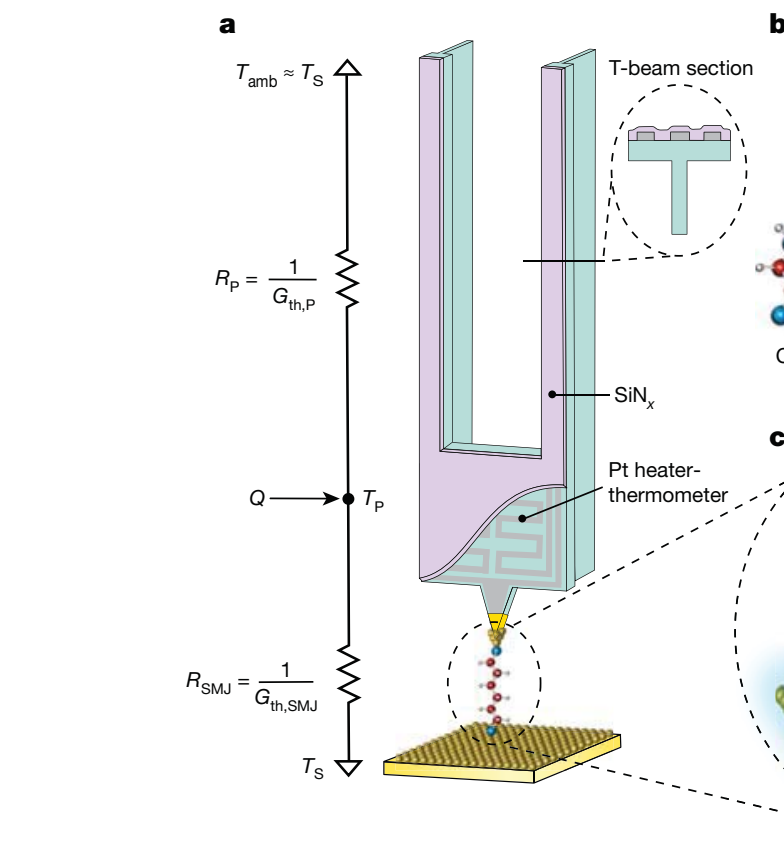

b

Fig. 1 Experimental set-up and strategy for quantifying heat transport in single-molecule junctions. a, Schematic of the calorimetric scanning thermal microscopy (C-SThM) set-up. Right, a single molecule is trapped between an Au-coated tip of the C-SThM probe, which features ' $\mathrm{T}$ '-shaped silicon nitride $\left(\mathrm{SiN}_{x}\right)$ beams and is heated to temperature $T_{\mathrm{P}}$ by input of a heat current $(Q)$ via an embedded serpentine Pt heater-thermometer, and an Au substrate at temperature $T_{\mathrm{S}}$ that is equal to ambient temperature $\left(T_{\mathrm{amb}}\right)$. The thermal conductance of single-molecule junctions is quantified by recording the temperature change of the Pt heater-thermometer when a single-molecule junction is broken. Left, resistance network capturing the thermal resistances of the molecular junction $\left(R_{\mathrm{SMJ}}=1 / G_{\mathrm{th}, \mathrm{SMJ}}\right)$ and the scanning probe $\left(R_{\mathrm{P}}=1 / G_{\mathrm{th}, \mathrm{P}}\right)$. $\mathbf{b}$, Schematics of the alkanedithiol
- d

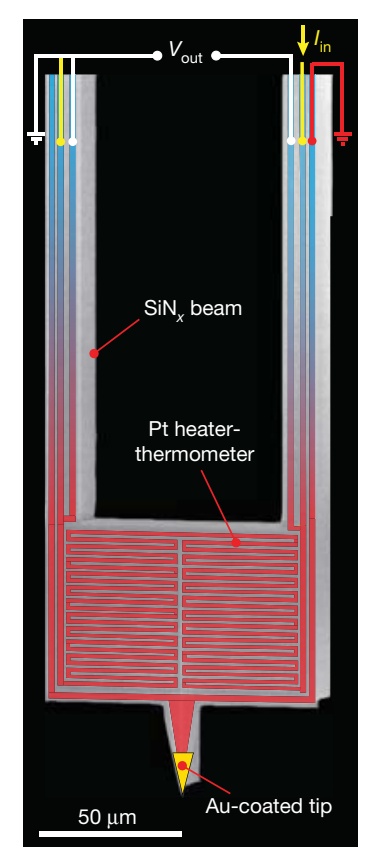

molecules (Cn) studied in this work; $n=2,4,6,8,10$ denotes the number of carbon atoms in the molecules (red, carbon atom; grey, hydrogen atom; blue, sulphur atom). c, Magnified view of ringed area in a, describing the trapping of a single C6 molecule between the heated Au STM tip and the cold Au substrate. d, Scanning electron microscope image (false coloured to highlight the Pt heater-thermometer) of a custom-fabricated C-SThM probe (which shows the tip end), featuring two long ' $\mathrm{T}$ '-shaped $\mathrm{SiN}_{x}$ beams (see beam cross-section shown ringed in a) and a serpentine Pt heater-thermometer integrated on a suspended micro-island. The electrical resistance of the Pt heater-thermometer is monitored by measuring the voltage output $\left(V_{\text {out }}\right)$ in the presence of an input d.c. current $\left(I_{\text {in }}\right)$.

${ }^{1}$ Department of Mechanical Engineering, University of Michigan, Ann Arbor, MI, USA. ${ }^{2}$ Department of Chemistry, Kookmin University, Seoul, South Korea. ${ }^{3}$ Okinawa Institute of Science and Technology Graduate University, Onna-son, Okinawa, Japan. ${ }^{4}$ Department of Physics, University of Konstanz, Konstanz, Germany. ${ }^{5}$ Department of Materials Science and Engineering, University of Michigan, Ann Arbor, MI, USA. ${ }^{6}$ Present address: Smalley-Curl Institute and Department of Physics and Astronomy, Rice University, Houston, TX, USA. ${ }^{7}$ Present address: Department of Energy Engineering, Ulsan National Institute of Science and Technology (UNIST), Ulsan, South Korea.*e-mail: fabian.pauly@oist.jp; syjang@kookmin.ac.kr; pramodr@umich.edu; meyhofer@umich.edu 


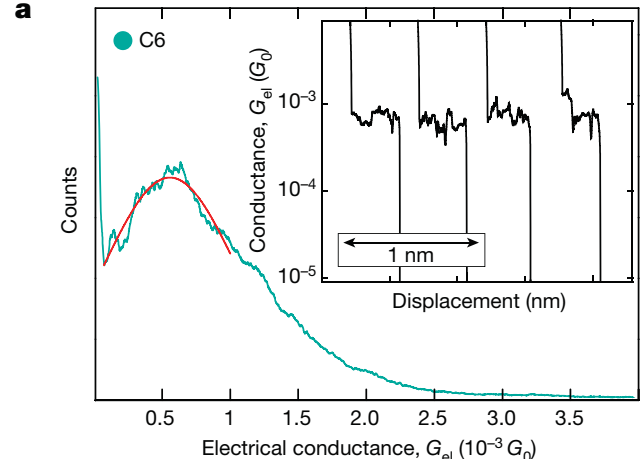

b

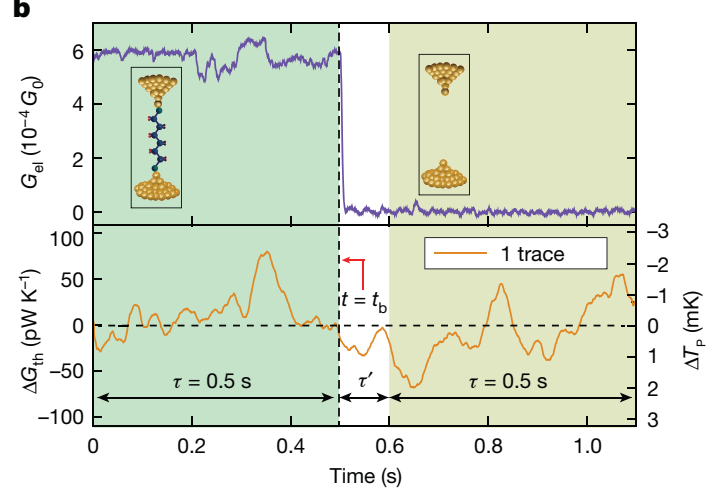

Fig. 2 Measurement of electrical and thermal conductance of Au-C6-Au single-molecule junctions. a, Main panel, histogram (shown in teal) of the electrical conductance of Au-C6-Au junctions obtained from approximately 500 independent traces of electrical conductance versus displacement. Inset, representative traces of the electrical conductance for four independent measurements. A Gaussian fit to the histogram peak is represented by the solid red line. b. Experimental protocol for measuring the thermal conductance of a single-C6 junction (see Methods for details). Upper panel, the electrical conductance trace indicates rupture (at time $t=t_{\mathrm{b}}$ ) of a single-molecule junction by a sudden drop of the measured $G_{\mathrm{el}}$ value. Lower panel, the coincident thermal conductance change ( $\Delta G_{\mathrm{th}}$, left axis) and the related temperature change

necessary to develop single-molecule measurement techniques ${ }^{1,3,5,7,9}$ to avoid the confounding effects of ensemble measurements-including uncertainties in the actual number of molecules contributing to transport through the junctions and the effects of intermolecular interactions - and to study systematically the electrical conduction properties of genuine single-molecule entities. Corresponding efforts over the past decade have been made to experimentally characterize heat transport in ensembles of molecules such as self-assembled monolayers ${ }^{22-24}$ and polymer nanofibres ${ }^{18,25}$. Not surprisingly, these thermal ensemble measurements face challenges and uncertainties similar to those found in previous monolayer electrical measurements, and intermolecular interactions are expected to have an influence on the thermal transport properties of molecular junctions ${ }^{16,26}$. Although recent experimental advances ${ }^{12,27}$ have enabled heat transport studies in metallic single-atom junctions (where thermal conductances are in the region of $500 \mathrm{pW} \mathrm{K}^{-1}$ ), similar endeavours for single-molecule junctions-where contributions to heat transport by electrons are negligible and heat flow is instead dominated by phonons resulting in low thermal conductance values (tens of picowatts per kelvin)-have remained unattainable owing to experimental challenges in detecting such small conductances. This situation is especially frustrating as computations have predicted several interesting thermal transport properties in one-dimensional molecular and polymer junctions ${ }^{13-16}$.

The first measurements of thermal transport in single-molecule junctions we report here are enabled by our custom-developed scanning probe technique, called calorimetric scanning thermal microscopy $(\mathrm{C}-\mathrm{SThM})^{12}$, which has excellent mechanical stability and ultra-high thermal sensitivity. The nanofabricated C-SThM probes

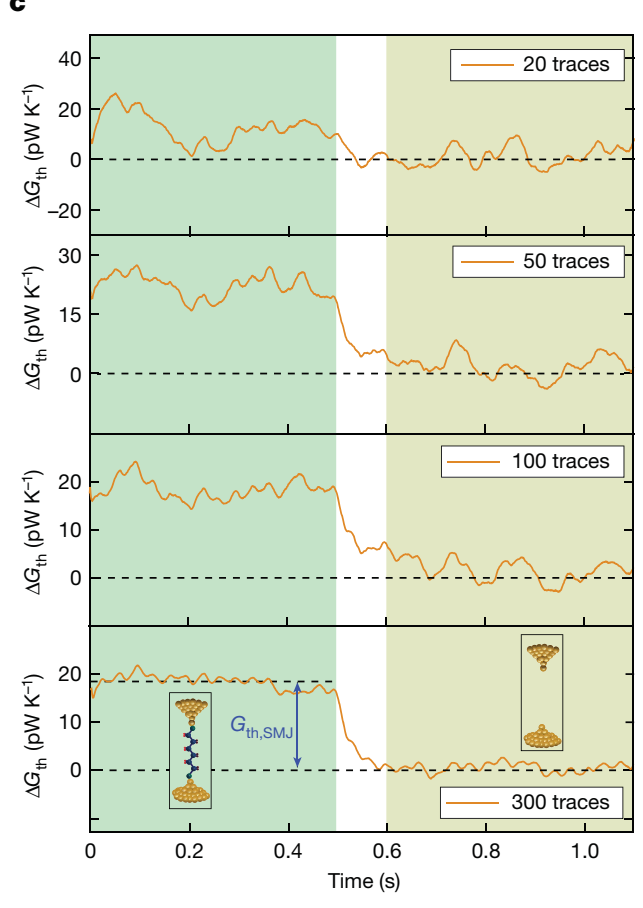

of the probe ( $\Delta T_{\mathrm{P}}$, right axis), where the small effects of Joule heating are already accounted for (see Methods). It can be seen that, unlike the clearly identifiable electrical conductance change associated with the breaking of the junction, the corresponding thermal conductance change is not discernible in the noisy signal. $\mathbf{c}$, An improved signalto-noise ratio is obtained upon aligning via $G_{\mathrm{el}}$ and averaging multiple thermal conductance traces. $G_{\mathrm{th}, \mathrm{SM}}$, indicated by the drop in the thermal conductance signal after $0.5 \mathrm{~s}$, can be seen after averaging 50 traces and is about $18 \mathrm{pW} \mathrm{K}^{-1}$ for $\mathrm{Au}-\mathrm{C} 6-\mathrm{Au}$ single-molecule junctions. The coloured regions in $\mathbf{b}$ and $\mathbf{c}$ with their insets indicate the discernible pre- and post-rupture portions of the recorded and averaged traces.

(see Extended Data Fig. 1 for the detailed fabrication process) feature a suspended micro-island supported by two thin, long ' $\mathrm{T}$ '-shaped silicon nitride $\left(\mathrm{SiN}_{x}\right)$ beams with both very high stiffness $\left(>10^{4} \mathrm{~N} \mathrm{~m}^{-1}\right.$ in the normal direction, see Methods) and very small thermal conductance $\left(G_{\mathrm{th}, \mathrm{P}} \approx 800 \mathrm{nW} \mathrm{K}{ }^{-1}\right.$; here and elsewhere, subscript $\mathrm{P}$ indicates that a property of the probe is being given). A platinum (Pt) resistor of serpentine geometry is embedded into the micro-island and serves as both a heater and a highly sensitive resistance thermometer. When combined with the time-averaging scheme described below and in Methods, it reaches a temperature resolution of about $0.1 \mathrm{mK}$ that enables us to detect heat currents with a resolution of approximately $80 \mathrm{pW}$, or thermal conductance with a resolution of about $2 \mathrm{pW} \mathrm{K}$ root mean square (see Methods).

Figure 1a depicts the experimental set-up and the basic strategy for quantifying thermal conductance at the single-molecule level. The C-SThM probe, located in an ultra-high-vacuum (UHV) environment, is heated above ambient to a temperature $T_{\mathrm{P}}$, typically $320-340 \mathrm{~K}$, by supplying a constant electric current (about 30-40 $\mu \mathrm{A}$ ) to the serpentine Pt resistor. The Au substrate, located in the same UHV environment, is connected to a thermal reservoir maintained at ambient room temperature $T_{\mathrm{S}}=295 \mathrm{~K}$ ( $\mathrm{S}$ indicates the Au substrate). The planar surface of the Au substrate is coated with a self-assembled monolayer of prototypical thiol-terminated alkane molecules that are widely regarded as a model system and have been extensively explored computationally ${ }^{13,15}$. We first create molecular junctions by displacing the $\mathrm{Au}$-coated scanning probe tip at a constant speed via piezoelectric actuation towards the Au substrate until contact is made between the two Au electrodes. With a voltage bias applied between the Au tip and 

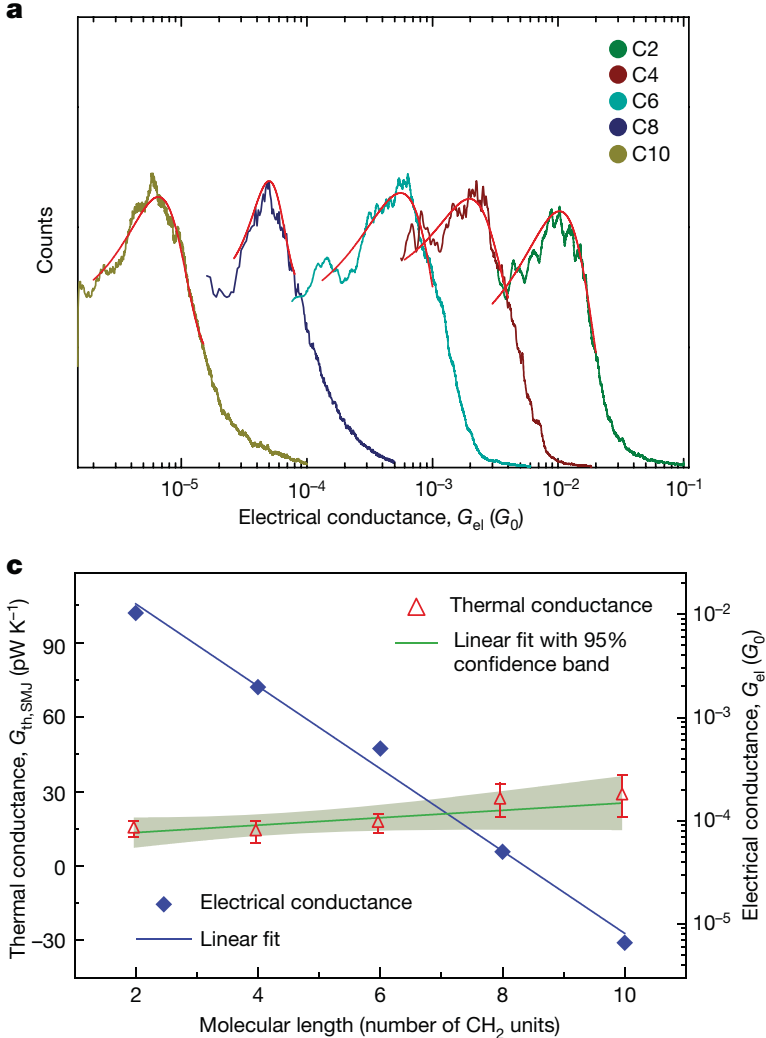

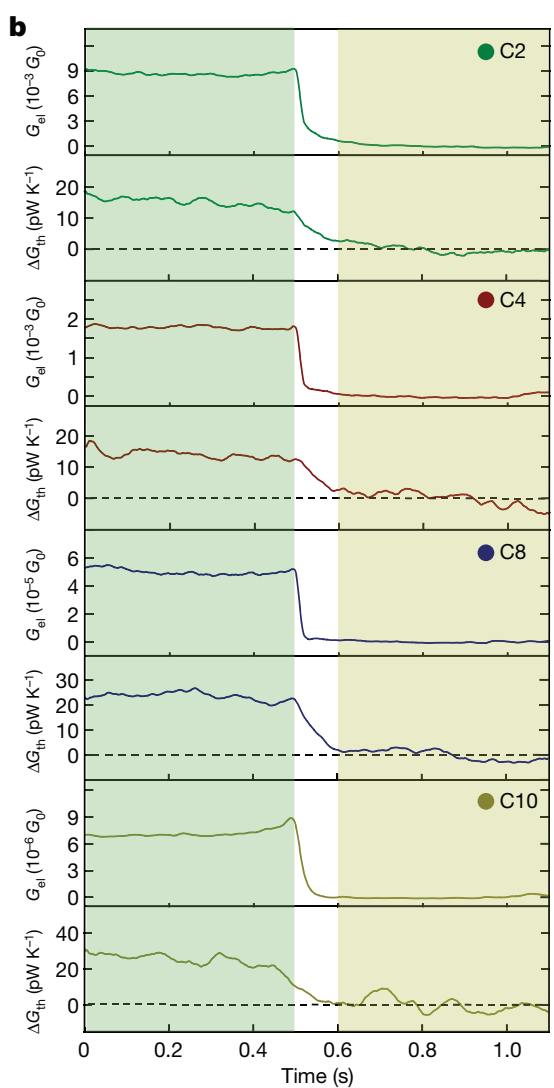

conductance (red triangles, left axis) as a function of the molecular length, as given by the number of $\mathrm{CH}_{2}$ units in the alkanedithiol junctions. The solid blue line indicates a linear fit to the electrical conductance data on a logarithmic scale. The measured thermal conductance data are fitted by a linear curve (green line) on a linear scale, with the region shaded in light green representing the $95 \%$ confidence band. Error bars represent one standard deviation of the data obtained from three sets of measurements for each molecule.

of $\mathrm{Au}-\mathrm{C} 6-\mathrm{Au}$ junctions is close to (within one standard deviation around the Gaussian-fitted histogram peak) the most probable low-bias conductance, and monitor the electrical current and temperature of the probe until the molecular junction spontaneously breaks. The top panel in Fig. $2 b$ shows a typical electrical conductance trace measured for an $\mathrm{Au}-\mathrm{C} 6-\mathrm{Au}$ single-molecule junction, showing how the electrical conductance suddenly drops within a few milliseconds (the time constant of the electrical measurements) when the molecular junction breaks. As the Joule heating is small for Au-C6-Au junctions (see Methods) and breaking removes the thermal conduction pathway through the molecular junction, we expect a small temperature rise in the probe $\left(\Delta T_{\mathrm{P}}\right)$ immediately after the junction is broken. This temperature change can be related to the change in the thermal conductance of the junction $\left(\Delta G_{\mathrm{th}}\right)$, that is, the thermal conductance of a single-molecule junction $\left(G_{\mathrm{th}, \mathrm{SMJ}}\right)$, via $G_{\mathrm{th}, \mathrm{SMJ}}=-\Delta G_{\mathrm{th}} \approx G_{\mathrm{th}, \mathrm{P}} \Delta T_{\mathrm{P}} /\left(T_{\mathrm{P}}-T_{\mathrm{S}}\right)$ (see Methods), where $G_{\mathrm{th}, \mathrm{P}}$ is the thermal conductance of the probe and $T_{\mathrm{P}}-T_{\mathrm{S}}$ is the temperature difference between the probe and the substrate. The bottom panel of Fig. $2 \mathrm{~b}$ presents the measured temperature change of the probe (right $y$-axis), from which the thermal conductance, $\Delta G_{\text {th }}$ (left $y$-axis), can be directly determined. (See Methods for a description of how the measured temperature change of the probe is processed and how the effects of Joule heating, which are small in this case but increase for shorter molecules, are systematically accounted for.)

Because the thermal conductance of the $\mathrm{Au}-\mathrm{C} 6-\mathrm{Au}$ singlemolecule junction is small relative to the noise present in $\Delta T_{\mathrm{B}}$ preventing reliable direct detection of changes in thermal conductance, we applied an averaging scheme to improve the signal-to-noise ratio of the thermal we stop the tip withdrawal process when the electrical conductance 


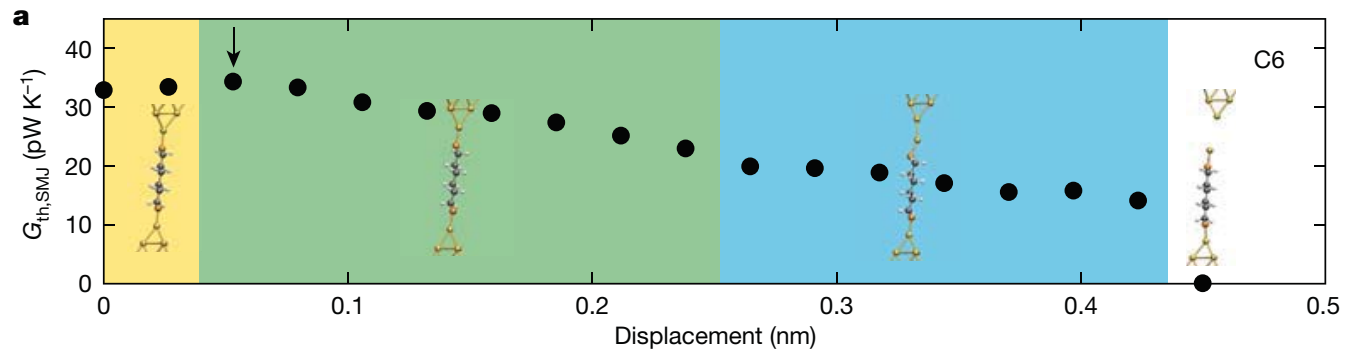

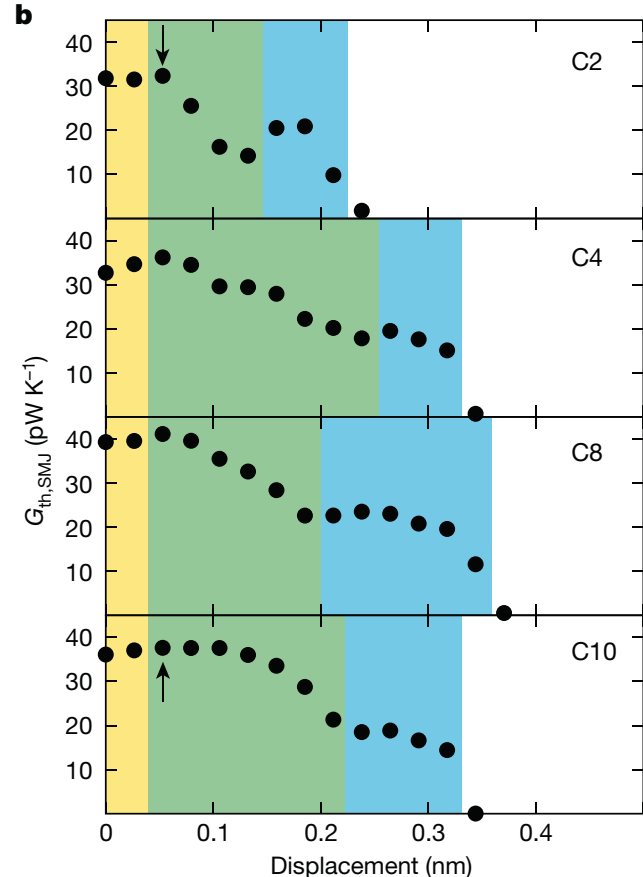

Fig. 4 | First-principles calculations of the thermal transport through alkanedithiol single-molecule junctions. a, Calculated thermal conductance as a function of electrode displacement for an $\mathrm{Au}-\mathrm{C} 6-\mathrm{Au}$ single-molecule junction. Different regions of junction stretching are distinguished by differently coloured backgrounds, and characterized by the representative geometries shown as insets: plateau (yellow), decay (green), pulled-out gold atoms (blue) and broken junction (white).

b. Thermal conductance as a function of electrode displacement for $\mathrm{C} 2$, $\mathrm{C} 4, \mathrm{C} 8$ and C10. c, Mean thermal conductance as calculated from the green area ( $\mathrm{AG}$, green triangles) and the blue area (AB, blue triangles). Error bars show maximum and minimum thermal conductances in the

measurements and resolve $\Delta G_{\text {th }}$. In brief, we performed many measurements (hundreds) following the protocol described above, and first used the electrical conductance versus time traces to identify the time point $t_{\mathrm{b}}$ when the single-molecule junction breaks $\left(t_{\mathrm{b}}=0.5 \mathrm{~s}\right.$ in Fig. 2b). Using the electrical signal as a reference, thermal signals were then aligned and averaged (see Methods). As the averages over 20, 50, 100 and 300 traces in Fig. 2c illustrate, averaging suppresses noise and reveals a clear thermal conductance change $\left(\Delta G_{\text {th }}\right)$ that coincides with the electrical conductance change caused by the breaking of the single-molecule junction. This approach reveals a change in the conductance of about $18 \mathrm{pW} \mathrm{K}^{-1}$, which represents the thermal conductance of the Au-C6-Au single-molecule junction $\left(G_{\text {th,SMJ }}\right)$. In contrast to the rapid transition of the electrical signal on the breaking of the junction, the roll-off of the thermal conductance is much slower because it is limited by the thermal time constant of the scanning probe (about $25 \mathrm{~ms}$ ).

The ability to resolve the thermal conductance at the single-molecule scale offers unique opportunities to address fundamental questions ${ }^{13,15}$ with regard to how thermal transport in single-molecule junctions depends on molecular characteristics. We illustrate this with additional thermal transport measurements on a series of alkanedithiol

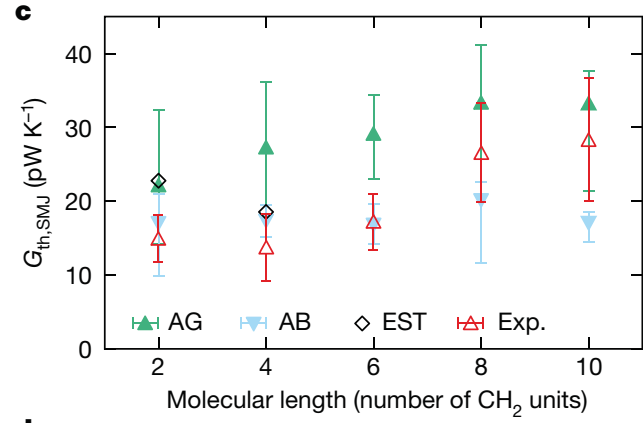

d

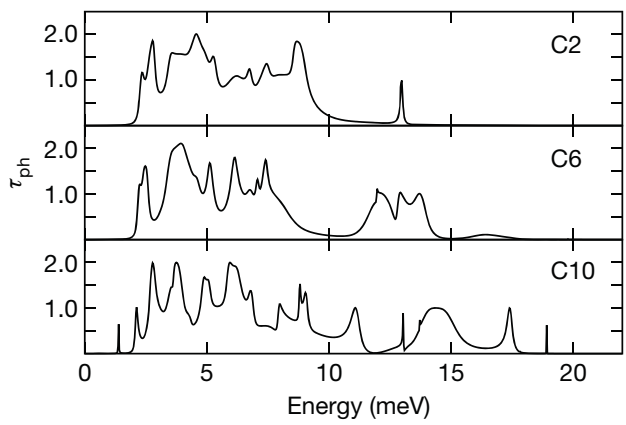

respective coloured regions. Estimates (EST, open diamonds) for the thermal conductance of $\mathrm{C} 2$ and $\mathrm{C} 4$ are obtained by taking the electronic contribution into account via the Wiedemann-Franz law and adding it to the corresponding average of the $\mathrm{AB}$ data points. The experimental data from Fig. $3 \mathrm{c}$ is also shown (red triangles and red error bars) to facilitate comparison between experiment and computations. d, Phonon transmission as a function of energy for $\mathrm{C} 2, \mathrm{C} 6$ and $\mathrm{C} 10$ junctions. The respective junction geometry, for which the transmission plot is performed, is indicated by an arrow in the corresponding trace in a or b. In each case the first geometry in the green region was selected.

molecules differing in the number of $\mathrm{CH}_{2}$ units (from 2 to 10 , with these molecules referred to as $\mathrm{C} 2$ to $\mathrm{C} 10$, respectively), to explore the influence of molecular length. Figure 3 a shows the measured electrical conductance histograms for the studied molecules, with the Gaussianfitted peak values summarized in Fig. 3c. The data document an exponential decay of the electrical conductance $\left(G_{\mathrm{el}}\right)$ of single-alkanedithiol junctions with increasing molecular length $(L)$, indicating tunnelling-dominated electron transport. We extract a tunnelling decay constant $\left(\beta\right.$, where $\left.G_{\mathrm{el}} / G_{0} \propto \mathrm{e}^{-\beta L}\right)$ of $0.92 \pm 0.05$ per $\mathrm{CH}_{2}$ unit, which agrees well with past work ${ }^{3}$. The measured thermal conductance of the single-molecule junctions containing $\mathrm{C} 2$ to $\mathrm{C} 10$ is shown in Fig. $3 \mathrm{~b}$, and the summary of the thermal conductance values is included in Fig. 3c. We note that, for all molecular junctions, the effect of Joule heating is systematically accounted for (see Methods). In strong contrast to the measured length-dependent electrical conductance, the thermal conductance of the single-alkanedithiol junctions exhibits a nearly length-independent behaviour with a value of approximately $20 \mathrm{pW} \mathrm{K}^{-1}$, suggesting that thermal transport in single-molecule junctions is ballistic.

To elucidate the microscopic origin of our observations, we use the Landauer-Büttiker formalism for coherent transport ${ }^{19,29}$ (see Methods). 
Combining non-equilibrium Green's function techniques with density functional theory (DFT) in a custom-developed code ${ }^{30,31}$, we compute, $\mathrm{ab}$ initio and thus without recourse to free parameters, the thermal conductance due to phonons for individual junction geometries matching the various alkane chain lengths and conditions used in our measurements. Figure 4 a shows the computed thermal conductance data for a C6 single-molecule junction as a function of electrode displacement, with the conductance-distance trace divided into different stages. The first stage, shaded in yellow, corresponds to a plateau as the molecule rotates slightly upon stretching with little change to the thermal conductance. In the second stage, shaded in green, the thermal conductance decreases as the junction is elongated due to $\mathrm{S}-\mathrm{Au}$ bond stretching and reconfigurations in the Au electrodes, which give rise to decreased metal-molecule coupling. Before the contact breaks, a third stage occurs, shaded in blue, where gold atoms are further pulled out of the electrodes and short atomic dimer chains form. This behaviour is well known in the context of atomic force studies $^{32}$ and typically leads to a small additional reduction of the phonon thermal conductance. In Fig. $4 \mathrm{~b}$ we depict corresponding traces for $\mathrm{C} 2, \mathrm{C} 4, \mathrm{C} 8$ and $\mathrm{C} 10$ junctions, all of which feature similar regions. In all cases, the junction breaks owing to the rupture of an $\mathrm{Au}-\mathrm{Au}$ bond (see also Extended Data Fig. 5).

The experimental data represent the thermal conductance at the point where the contact breaks, so we calculate for $\mathrm{C} 2$ to $\mathrm{C} 10$ the thermal conductance values $\mathrm{AG}$ and $\mathrm{AB}$ that are the average over the stretched junctions in the green- and blue-shaded regions in Fig. 4a, b, respectively, and compare these in Fig. $4 \mathrm{c}$ against the measured thermal conductance values. The computed $\mathrm{AB}$ and $\mathrm{AG}$ values lie in the range $16-21 \mathrm{pW} \mathrm{K}^{-1}$ and $22-33 \mathrm{pW} \mathrm{K}^{-1}$, respectively, and agree well with the measured data. Further, we observe that the computed phononic contribution to the thermal conductance of the junctions is nearly independent of molecular length. For completeness, we also estimate the electronic contribution to the thermal conductance using the measured electrical conductance (Fig. 3a) and the WiedemannFranz law (see Methods for more details). We find that the electronic contribution is about $5.7 \mathrm{pW} \mathrm{K}^{-1}$ and $1.1 \mathrm{pW} \mathrm{K}^{-1}$ for $\mathrm{C} 2$ and $\mathrm{C} 4$, respectively, while it is negligible for all other molecules. We have added these values (indicated by open diamonds) to the $A B$ data in Fig. 4c. On the whole, the theoretically determined thermal conductance values are in good agreement with the experimental data. We note that in contrast to previous studies ${ }^{15}$, where thermal transport was calculated for single-molecule junctions under minimal tension, our analysis here includes the effect of stretching and reveals a lower thermal conductance when a junction is close to rupture.

To understand further how heat is transported through single-molecule junctions, we show in Fig. 4d the computed energy-dependent transmission function $\tau_{\mathrm{ph}}(E)$ for $\mathrm{C} 2, \mathrm{C} 6$ and $\mathrm{C} 10$ junctions. The function quantifies the probability of elastic phonon transmission at a specific energy from one electrode to the other via the bridging molecule. Owing to coupling to the continuous modes of the metal electrodes, $\tau_{\mathrm{ph}}(E)$ shows broad resonances with positions and widths that depend on the precise contact geometry (see Methods and Extended Data Fig. 6 for further discussions). We notice that the transmission functions in Fig. $4 \mathrm{~d}$ are finite only in an energy range from 0 to $E_{\max } \approx 20 \mathrm{meV}$, where $E_{\max }$ represents the highest phonon energy of Au. At room temperature all the transmission resonances in this energy interval determine the actual value of the thermal conductance (see Methods section 'Computational methods', including equation (1), for further details), and they mainly arise from centre-of-mass motions of the molecule between the electrodes or low-energy molecular vibrations. For longer molecules more transmission resonances arise between 0 and $E_{\max }$, since more molecular modes overlap with the phonon density of states of $\mathrm{Au}$. In addition, we find that for all junctions the transmission values are below 3, which, as we have discussed before ${ }^{29}$, is related to the linear, one-dimensional structure of the alkane molecules. Last, we note that anharmonic effects, which result in phonon-phonon scattering, could reduce heat flow. However, owing to the long wavelengths of the vibrational modes relevant for thermal transport we expect such anharmonic effects to be small ${ }^{13}$.

Our experimental results illustrate a nearly length-independent thermal conductance in alkane-based single-molecule junctions, which is in strong contrast with the corresponding exponential length dependence of the electrical conductance. In contrast to work on monolayers and polymer bundles, our work realizes the long-sought goal of unambiguous identification of thermal conductance at the single-molecule level. Our ab initio computational analysis provides strong support for our experimental data regarding the length independence of thermal conductance, and offers mechanistic insights in terms of molecular vibrational properties. The experimental advances presented here will enable systematic studies of thermal transport through single-molecule junctions and other one-dimensional systems such as polymer chains, which are of great current interest but so far have remained experimentally inaccessible.

1. Smit, R. H. M. et al. Measurement of the conductance of a hydrogen molecule. Nature 419, 906-909 (2002)

2. Nitzan, A. \& Ratner, M. A. Electron transport in molecular wire junctions. Science 300, 1384-1389 (2003).

3. Xu, B. \& Tao, N. J. Measurement of single-molecule resistance by repeated formation of molecular junctions. Science 301, 1221-1223 (2003).

4. Reecht, G. et al. Electroluminescence of a polythiophene molecular wire suspended between a metallic surface and the tip of a scanning tunneling microscope. Phys. Rev. Lett. 112, 047403 (2014).

5. Reddy, P., Jang, S. Y., Segalman, R. A. \& Majumdar, A. Thermoelectricity in molecular junctions. Science 315, 1568-1571 (2007).

6. Cui, L. et al. Peltier cooling in molecular junctions. Nat. Nanotechnol. 13, 122-127 (2018).

7. Garner, M. H. et al. Comprehensive suppression of single-molecule conductance using destructive $\sigma$-interference. Nature 558, 415-419 (2018).

8. Miao, R. et al. Influence of quantum interference on the thermoelectric properties of molecular junctions. Nano Lett. 18, 5666-5672 (2018).

9. Lee, W. et al. Heat dissipation in atomic-scale junctions. Nature 498, 209-212 (2013).

10. Ward, D. R., Corley, D. A., Tour, J. M. \& Natelson, D. Vibrational and electronic heating in nanoscale junctions. Nat. Nanotechnol. 6, 33-38 (2011).

11. Lumbroso, O. S., Simine, L., Nitzan, A., Segal, D. \& Tal, O. Electronic noise due to temperature differences in atomic-scale junctions. Nature $562,240-244$ (2018).

12. Cui, L. et al. Quantized thermal transport in single-atom junctions. Science $\mathbf{3 5 5}$, 1192-1195 (2017).

13. Segal, D., Nitzan, A. \& Hänggi, P. Thermal conductance through molecular wires. J. Chem. Phys. 119, 6840-6855 (2003).

14. Sadeghi, H., Sangtarash, S. \& Lambert, C. J. Oligoyne molecular junctions for efficient room temperature thermoelectric power generation. Nano Lett. 15, 7467-7472 (2015).

15. Klöckner, J. C., Bürkle, M., Cuevas, J. C. \& Pauly, F. Length dependence of the thermal conductance of alkane-based single-molecule junctions: an ab initio study. Phys. Rev. B 94, 205425 (2016).

16. Henry, A. \& Chen, G. High thermal conductivity of single polyethylene chains using molecular dynamics simulations. Phys. Rev. Lett. 101, 235502 (2008).

17. Aradhya, S. V. \& Venkataraman, L. Single-molecule junctions beyond electronic transport. Nat. Nanotechnol. 8, 399-410 (2013).

18. Shen, S., Henry, A., Tong, J., Zheng, R. T. \& Chen, G. Polyethylene nanofibres with very high thermal conductivities. Nat. Nanotechnol. 5, 251-255 (2010).

19. Dubi, Y. \& Di Ventra, M. Heat flow and thermoelectricity in atomic and molecular junctions. Rev. Mod. Phys. 83, 131-155 (2011).

20. Xiang, D., Wang, X., Jia, C., Lee, T. \& Guo, X. Molecular-scale electronics: from concept to function. Chem. Rev. 116, 4318-4440 (2016)

21. Rincón-García, L., Evangeli, C., Rubio-Bollinger, G. \& Agraït, N. Thermopower measurements in molecular junctions. Chem. Soc. Rev. 45, 4285-4306 (2016).

22. Wang, R. Y., Segalman, R. A. \& Majumdar, A. Room temperature thermal conductance of alkanedithiol self-assembled monolayers. Appl. Phys. Lett. 89, 173113 (2006).

23. Wang, Z. et al. Ultrafast flash thermal conductance of molecular chains. Science 317, 787-790 (2007).

24. Meier, T. et al. Length-dependent thermal transport along molecular chains. Phys. Rev. Lett. 113, 060801 (2014). 
25. Wang, X., Ho, V., Segalman, R. A. \& Cahill, D. G. Thermal conductivity of high-modulus polymer fibers. Macromolecules 46, 4937-4943 (2013).

26. Majumdar, S., Malen, J. A. \& McGaughey, A. J. H. Cooperative molecular behavior enhances the thermal conductance of binary self-assembled monolayer junctions. Nano Lett. 17, 220-227 (2017).

27. Mosso, N. et al. Heat transport through atomic contacts. Nat. Nanotechnol. 12, 430-433 (2017)

28. Jang, S. Y., Reddy, P., Majumdar, A. \& Segalman, R. A. Interpretation of stochastic events in single molecule conductance measurements. Nano Lett. 6 , 2362-2367 (2006).

29. Klöckner, J. C., Cuevas, J. C. \& Pauly, F. Transmission eigenchannels for coherent phonon transport. Phys. Rev. B 97, 155432 (2018).
30. Pauly, F. et al. Cluster-based density-functional approach to quantum transport through molecular and atomic contacts. New J. Phys. 10, 125019 (2008).

31. Bürkle, M., Hellmuth, T. J., Pauly, F. \& Asai, Y. First-principles calculation of the thermoelectric figure of merit for [2,2]paracyclophane-based single-molecule junctions. Phys. Rev. B 91, 165419 (2015).

32. Krüger, D., Fuchs, H., Rousseau, R., Marx, D. \& Parrinello, M. Pulling monatomic gold wires with single molecules: an ab initio simulation. Phys. Rev. Lett. 89, 186402 (2002)

Publisher's note: Springer Nature remains neutral with regard to jurisdictional claims in published maps and institutional affiliations.

(C) The Author(s), under exclusive licence to Springer Nature Limited 2019 


\section{METHODS}

Nanofabrication of scanning thermal probes. To fabricate the probes (Extended Data Fig. 1), we start with a 500- $\mu$ m-thick double-sided silicon wafer and form an $18-\mu \mathrm{m}$-deep and $1-\mu \mathrm{m}$-wide trench on the silicon wafer via wet oxidation and deep reactive ion etching (DRIE). A 600-nm-thick silicon nitride $\left(\mathrm{SiN}_{x}\right)$ layer was deposited on both sides of the wafer via low pressure chemical vapour deposition (LPCVD) and the back side was patterned to facilitate etching using potassium hydroxide $(\mathrm{KOH})$ for releasing the probe in the last step. A sensitive thermometer was defined by patterning a $30-\mathrm{nm}$-thick and $1-\mu \mathrm{m}$-wide platinum $(\mathrm{Pt})$ serpentine line. The tip was fabricated by first depositing a $100-\mathrm{nm}$-thick platinum film. Subsequently, a 30-nm-thick $\mathrm{SiN}_{x}$ layer was deposited via plasma-enhanced chemical vapour deposition (PECVD) to protect the front side of the probe during $\mathrm{KOH}$ etching. Two shadow masks were introduced separately to deposit a sputtered 50-nm-thick $\mathrm{SiN}_{x}$ film on the serpentine-shaped Pt covered region and a 500 -nm-thick gold $(\mathrm{Au})$ layer on the tip.

Characterization of thermal, electrical and mechanical properties. Temperature coefficient of resistance (TCR). To measure the TCR of the Pt thermometer, a small a.c. current of amplitude $I_{f}=1 \mathrm{nA}$ at frequency $f=200 \mathrm{~Hz}$ was supplied to the embedded Pt serpentine line on the probe, and the resultant $1 f$ component of the voltage signal, $V_{f}$, was measured using a lock-in amplifier (SR830) in a fourprobe configuration. The temperature-dependent electric resistance defined as $R(T)=V_{f} / I_{f}$ was evaluated by varying the temperature of the probe inside a cryostat (Janis ST-100). A representative plot of the measured resistance of a scanning thermal probe as a function of temperature is shown in Extended Data Fig. 2a. The TCR can be obtained by using the slope of the best-linear-fit curve of the measured data points. At room temperature, the TCR was found to be $(1.45 \pm 0.01) \times 10^{-3} \mathrm{~K}^{-1}$.

Thermal time constant of the probe. The thermal time constant of the calorimetric scanning thermal probes was determined by applying a sinusoidal electrical current with constant amplitude $I_{f}$ at varying frequency $f$ to the Pt resistor. This current enables sinusoidal Joule heating of the suspended island, $Q_{2} f$, with an associated temperature fluctuation at $2 f$ and an amplitude of $\Delta T_{2 f}$. The $3 f$ component of the output voltage across the Pt resistor $V_{3 f}$ was recorded using a lock-in amplifier (SRS 830). $\Delta T_{2 f}$ can subsequently be determined according to the relation $\Delta T_{2 f}=2 V_{3 f} f\left(\alpha I_{f} R\right)$, where $\alpha$ is the measured TCR and $R$ is the electrical resistance of the Pt serpentine line. The measured $\Delta T_{2 f}$, which was normalized by the amplitude at the lowest frequency, is shown as a function of the heating frequency in Extended Data Fig. 2b. Note that the $-3 \mathrm{~dB}$ point (thermal cut-off frequency) is $\sim 7 \mathrm{~Hz}$, which can be used to determine the time constant of the probe from $\tau=\left(2 \pi f_{-3 \mathrm{~dB}}\right)^{-1} \approx 25 \mathrm{~ms}$. Thermal conductance of the probe. This was measured by applying a sinusoidal electrical current with fixed frequency $f$ and varying amplitude $I_{f}$ to the embedded Pt serpentine line ${ }^{12}$ of the probe, resulting in Joule heating. The magnitude of the heating power in the serpentine line can be calculated as $Q_{2 f}=I_{f}^{2} R / 2$, resulting in a corresponding temperature increase $\Delta T_{2 f}$ of the island (distal end of the probe). The heating frequency $2 f$ was chosen to be $1 \mathrm{~Hz}$ to ensure a full thermal response of the probe. Similar to the characterization of the thermal time constant, $\Delta T_{2 f}$ can be quantified by recording the $3 f$ component of the output voltage across the Pt line. Extended Data Fig. 2c displays the relationship between the amplitude of the measured temperature increase $\Delta T_{2 f}$ and the input heating power $Q_{2 f}$. The thermal conductance of the probe can then be obtained via $G_{\mathrm{th}, \mathrm{P}}=Q_{2 f} / \Delta T_{2 f}$, which is estimated to be about $800 \mathrm{nW} \mathrm{K}^{-1}$.

Mechanical stiffness of the probe. Simulations with the finite element method (FEM) were carried out using COMSOL Multiphysics (Solid Mechanics module, COMSOL) to estimate the stiffness of the thermal probes using the following boundary conditions: a force of $50 \mathrm{nN}$, either in the normal or the transverse direction, was applied at the end of the probe tip, while the other end of the $\operatorname{SiN}_{x}$ cantilevers was fixed. From the computed resultant displacement field, the stiffness of the probe was estimated to be $\sim 14,000 \mathrm{~N} \mathrm{~m}^{-1}$ in the normal direction, and $\sim 275 \mathrm{~N} \mathrm{~m}^{-1}$ and $\sim 12.5 \mathrm{~N} \mathrm{~m}^{-1}$ in the transverse directions, respectively (as shown in Extended Data Fig. 3a-c). In our experiments, the normal stiffness is found to be sufficiently large to form stable molecular junctions.

Temperature distribution on the probe. Temperature fields generated on the probe due to d.c. Joule heating or d.c. heat input to the tip were simulated using COMSOL (Joule Heating and Thermal Expansion module). A 10- $\mu$ A d.c. electrical current (Extended Data Fig. 3d) was supplied to the Pt line or a $10-\mu \mathrm{W}$ d.c. heat current was input at the tip (Extended Data Fig. 3e), while the ends of the $\mathrm{SiN}_{x}$ cantilevers were held at $300 \mathrm{~K}$. We note that in both cases the Pt thermometer embedded in the island exhibits a uniform temperature distribution and the temperature drop occurs primarily along the beams.

Experimental set-up and measurement schemes. Ultra-low-noise measurement environment. All electrical and thermal measurements of single-molecule junctions were performed in a UHV $\left(\sim 10^{-9}\right.$ torr $)$ scanning probe instrument (RHK UHV 750), which is housed in a test chamber of a low-noise facility where the mechanical floor vibrations are maintained below the NIST-A standard.
The temperature drift of the chamber was actively controlled to vary below $100 \mathrm{mK}$ around a chosen set point at $295 \mathrm{~K}$.

Molecular sample preparation and cleaning protocol for probes. To facilitate the formation of single-molecule junctions during the experiments, self-assembled monolayers of alkanedithiol molecules were prepared on an ultra-flat planar $\mathrm{Au}$-coated substrate, which was prepared via template-stripping. The Au-coated substrate was immersed in $500-\mu \mathrm{M}$ ethanol solutions of alkanedithiol molecules (C2, C4, C6, C8, C10, from Sigma Aldrich with purity $>95 \%)$ to initiate the self-assembly process of the molecules on the Au surface. After $\sim 12 \mathrm{~h}$ of incubation, the samples were thoroughly rinsed in ethanol and dried in a nitrogen-filled glove box before being transferred into the UHV measurement environment. Furthermore, in order to ensure high cleanliness of the Au-coated scanning thermal probes, which is critical for successful thermal transport measurements, we followed a protocol reported elsewhere ${ }^{33}$. We note that it is critical to avoid any direct contact of the probe with the ambient, and multiple cycles of wet and plasma cleaning are usually needed to eliminate any detectable contamination on the probe. Formation of single-molecule junctions and transport measurement circuitry. All the single-molecule junctions were created between the scanning thermal probes and molecule-covered Au substrates. During the measurement, the probe was controllably displaced towards the substrate at a speed of $1 \mathrm{~nm} \mathrm{~s}^{-1}$, and withdrawn from the substrate at $0.05 \mathrm{~nm} \mathrm{~s}^{-1}$ after making contact as indicated by a sufficiently large electrical conductance (compared to the single-molecule conductance). The withdrawal of the scanning probe was stopped once a single-molecule junction was formed, as indicated by an approximately constant electrical conductance that was within one standard deviation of the single-molecule conductance obtained from the conductance histogram. Simultaneous electrical and thermal conductance measurements were recorded for a constant electrode separation until the particular singlemolecule junction spontaneously broke. The process of formation and breakdown of single-molecule junctions was repeated several hundred times for each type of molecule.

The electrical conductance was measured by supplying a d.c. voltage bias ( $30 \mathrm{mV}, 50 \mathrm{mV}, 100 \mathrm{mV}, 100 \mathrm{mV}$ and $200 \mathrm{mV}$ for C2-C10 junctions, respectively) across the scanning thermal probe and the Au substrate, while monitoring the tunnelling current across the junctions via a current amplifier (SR570). We note that the filter settings of the current amplifier resulted in an electrical time constant of $\sim 2 \mathrm{~ms}$ in all our experiments (see Figs. $2 \mathrm{~b}$ and $3 \mathrm{~b}$ ). Smaller voltage biases were chosen for shorter molecular junctions, which feature larger electrical conductances, to minimize the effects of Joule heating.

In order to study the thermal conductance of the junctions, the temperature change of the scanning thermal probe was measured before and after the breakdown of the single-molecule junctions. For this purpose we monitored the change in the electric resistance of the embedded Pt resistance thermometer via a half-Wheatstone bridge. The output voltage signal of the bridge circuit in the presence of a d.c. electric current was first amplified by an instrumentation amplifier (AD524) with a gain of 100 and subsequently measured using a low-noise voltage amplifier (SR 560 with a gain of 100).

Selection criteria for single-molecule traces. To analyse the thermal conductance of single-molecule junctions, we identify single-molecule events via off-line analysis from our continuous recordings by applying the following criteria: (1) the electrical conductance drops in a clear last step from a constant, expected electrical conductance value (corresponding to the previously established values, see above), signalling the presence of a single-molecule junction before breakdown; (2) during formation and following breakdown of the junction, the thermal conductance from the probe to the monolayer sample is relatively stable (drift $<100 \mathrm{pW} \mathrm{K}^{-1}$ in $0.5 \mathrm{~s}$ ), signifying a thermal measurement that is not compromised by a large change of background conduction pathways. The first criterion ensures that we are only employing data from single-molecule junctions with well-defined electrical conductance corresponding to the most probable value, as identified from the analysis of the conductance histogram (Figs. 2a and 3a). The second criterion is principally informed by our past work ${ }^{33}$, which suggests that the presence of organic contamination leads to parasitic conductances at the sub-nanowatt per K to nanowatt per $\mathrm{K}$ level. The variation in this background conductance as a function of time, if large, can limit the resolution of our time-averaging approach. In our analysis we found that drift values $<100 \mathrm{pW} \mathrm{K}^{-1}$ in a 0.5 -s period after rupture of contact are sufficient to achieve the desired signal-to-noise ratio. Rigorous application of the above criteria avoids artefacts and ensures reliable single-molecule thermal conductance measurements. A majority ( $>90 \%$ ) of the curves that satisfy the first criterion were also found to satisfy the second. In the relatively rare events $(<10 \%)$ where large background drift was observed, we deemed the experiment to have failed and excluded the curve from the thermal conductance analysis using a completely automated process.

Noise reduction due to the time-averaging scheme. The measured temperature change of the scanning thermal probe is associated with substantial noise contributions from electronics (amplifiers), Johnson noise, shot noise and temperature drift of the measurement environment. As shown in Fig. 2b, the unprocessed 
thermal signals are featureless and buried in large noise $\left(\sim 100 \mathrm{pW} \mathrm{K}^{-1}\right)$. To improve the signal-to-noise ratio of the measurements, a time-averaging scheme is applied to the thermal conductance traces, which were acquired through independent measurements of many molecular junctions $(\sim 100)$. Briefly, the time at which a single-molecule junction breaks $\left(t_{\mathrm{b}}\right)$ was first detected by analysing the time series of electrical conductance traces. Subsequently the $\Delta G_{\text {th }}$ signal corresponding to the same electrical conductance trace was demarcated into a $\tau=0.5 \mathrm{~s}$ region (shaded in green in Fig. 2b, bottom panel) before $t_{\mathrm{b}}$, and two intervals $\tau^{\prime}=0.1 \mathrm{~s}$ (unshaded) and $\tau=0.5 \mathrm{~s}$ (shaded in brown) after $t_{\mathrm{b}}$. We note that the $\tau^{\prime}=0.1 \mathrm{~s}$ interval (around four times the thermal time constant of the probe) corresponds to the time required to achieve the full thermal response of the probe after the junction-breaking event. Further, the average value of the $\Delta G_{\mathrm{th}}$ signal in the subsequent $0.5 \mathrm{~s}$ after breaking of the molecular junction is averaged and set to zero by suitably offsetting the curve. (This procedure ensures compliance with the physical expectation that the thermal conductance change after breaking of the junction is negligibly small.) Finally, the thermal conductance traces from each of the individual experiments were aligned using $t_{\mathrm{b}}$ as the reference point, and data from corresponding time points were averaged. Following a procedure described in detail in our previous work ${ }^{9}$, we can estimate the smallest thermal conductance change $\left(\Delta G_{\mathrm{th}, \mathrm{min}}\right)$ detectable using our time-averaging scheme to be:

$$
\begin{aligned}
& \Delta G_{\text {th, } \min } \approx \frac{G_{\text {th }, \mathrm{P}}}{T_{\mathrm{P}}-T_{\mathrm{S}}} \frac{1}{I R_{0} \alpha} \\
& \times\left\{\int_{-\infty}^{+\infty} G_{\text {noise }}(f)\left[\frac{2 \tau+\tau^{\prime}}{\tau} \frac{\sin (2 \pi f \widetilde{T})}{2 \pi f \widetilde{T}} \frac{2 \sin \left[\pi f\left(\tau+\tau^{\prime}\right)\right] \sin (\pi f \tau)}{\sin \left[\pi f\left(2 \tau+\tau^{\prime}\right)\right]}\right]^{2} \mathrm{~d} f\right\}^{1 / 2}
\end{aligned}
$$

Here $G_{\text {noise }}(f)$ is the power spectral density at frequency $f$ associated with the temperature noise that the probe is subject to, and $2 \widetilde{T}$ is the total time over which the averaging is performed. For example, for 100 molecular junctions the total averaging time $2 \widehat{T}$ equals $110 \mathrm{~s}$. By following the protocol that we have developed previously $^{34}$ to measure the noise spectrum of a Pt resistance thermometer, we can estimate the power spectral density. With this information and the above equation, we estimate $\Delta G_{\text {th,min }}$ to be $\sim 2 \mathrm{pW} \mathrm{K}^{-1}$. Finally, we note that the electrical conductance traces show additional stepwise changes before rupture of the last junction (see Extended Data Fig. 4 top panels) that represent recordings during the withdrawal of the tip from multi-molecule junctions. These additional changes do not yield identifiable multiple conductance states in electrical conductance histograms (see, for example, Fig. 2a). Further, given the low thermal conductance of the studied molecular junctions, the thermal traces of multi-molecule junctions are, as expected, largely featureless (see Extended Data Fig. 4, bottom panels). An averaging approach analogous to the successful analysis of the thermal conductance of single-molecule junctions cannot yield the corresponding thermal conductances of these multiplemolecule junctions, as these states are not well correlated in time and step-size compared to the single-molecule junction states.

Effect of Joule heating on measurements of the thermal conductance. In addition to averaging the signals from many individual thermal conductance events as discussed above, we need to account for the heat dissipation that results from the applied electrical bias. Specifically, when a voltage bias $(V)$ is supplied across a junction of resistance $R$, it results in a total heat dissipation of $V^{2} / R$. Since the Seebeck coefficient of alkanedithiol junctions is very small ${ }^{35}$, the heat dissipation in the electrodes is symmetric to an excellent approximation ${ }^{9}$. Therefore, the heat dissipated in the probe due to the voltage bias is given by $V^{2} / 2 R$. When the single-molecule junction is broken, the probe not only heats up due to the loss of a thermal conduction pathway, but there is also a competing effect that attenuates the temperature drop as the heat dissipation in the probe decreases by $V^{2} / 2 R$. In order to systematically account for this effect, we add $\Delta T_{\text {Joule }}=V^{2} /\left(2 R G_{\text {th }, \mathrm{P}}\right)$ to the measured data in the range 0 to $t_{\mathrm{b}}$ seconds (that is, for the region before the junction is broken) to obtain the $\Delta T_{\mathrm{P}}$ plot in Fig. 2b and all other related $\Delta G_{\text {th }}$ plots shown in the manuscript (Figs. 2c, 3b). These corrections are modest for $\mathrm{C} 6$ $(\sim 20 \%), \mathrm{C} 8(<2 \%)$ and $\mathrm{C} 10(<2 \%)$ junctions, but they are sizable for C2 $(\sim 60 \%)$ and $\mathrm{C} 4(\sim 30 \%)$ junctions. Here, all the percentages represent how large $\Delta T_{\text {Joule }}$ is with respect to the observed temperature drop $\Delta T_{\mathrm{P}}$ when the junction breaks. Effect of intermolecular interactions on the measurements of the thermal conductance. Given the fact that all our experiments are performed in a UHV environment, the probability of interaction between the single-molecule junction of interest and other molecules is much smaller than that in experiments performed in a solution environment, where several surrounding molecules can potentially interact with the junction. Further, it is to be noted that in our experiments the single-molecule junction is expected to be isolated from surrounding molecules as the junction is created between elongated $\mathrm{Au}$ chains that protrude out from the electrodes. Therefore, the probability of interaction with surrounding molecules is expected to be very low.
Influence of near-field radiative heat transfer. Near-field radiative heat transfer has a negligible impact on our measurements. In particular, our strategy for determining the thermal conductance of a single-molecule junction relies on measuring the change of the thermal conductance when a single molecule that is bridging the calorimeter and the substrate breaks away. In the absence of any drift in the gap size, the near-field contribution before and after the junction-breaking event is identical. For this reason, near-field thermal radiation makes no contribution to the measurement, as we are determining the change in the thermal conductance upon the breakdown of the junction. The drift in the gap size of our system of $<1 \AA \mathrm{min}^{-1}$ translates to $<15 \mathrm{pm}$ in a 1-s time interval. Given our past analysis ${ }^{33}$, the near-field radiative conductance change is expected to be $\sim 2 \mathrm{pW} \mathrm{K}^{-1}$ when the gap size changes by $\sim 1 \mathrm{~nm}$. Therefore, the change in the near-field contribution due to gap size drift of $\sim 15 \mathrm{pm}$ is negligibly small $\left(<0.03 \mathrm{pW} \mathrm{K}^{-1}\right)$ when compared to the thermal conductance of a single-molecule junction $\left(\sim 20 \mathrm{pW} \mathrm{K}^{-1}\right)$.

Computational methods. Thermal conductance within the Landauer-Büttiker approach. To calculate the thermal conductance of molecular junctions, we employ the Landauer-Büttiker formalism for coherent transport ${ }^{19,29,36,37}$. This theory describes phonon transport as phase-coherent and elastic. Since in this formalism transport is described as a scattering problem of waves, the key quantity is the probability $\tau_{\mathrm{ph}}(E)$ of a phonon at a given energy $E$ to be transmitted from one lead to the other, which is computed using the procedures developed in our past work ${ }^{15,29,31}$. The linear response coefficient $\left(G_{\mathrm{th}, \mathrm{SMJ}}\right)$ can be calculated using:

$$
G_{\mathrm{th}, \mathrm{SMJ}}=\frac{1}{h} \int_{0}^{\infty} E \tau_{\mathrm{ph}}(E) \frac{\partial n(E, T)}{\partial T} \mathrm{~d} E
$$

where $n(E, T)=\left[\exp \left(E / k_{\mathrm{B}} T\right)-1\right]^{-1}$ is the Bose function. The thermal conductance is given as an energy integral over the transmission function weighted by energy and the temperature derivative of the Bose function, which considers the energetic content of the transmitted phonons and the difference in phonon populations in the leads, respectively.

DFT modelling. In the calculation of the phononic transmission function, the dynamical matrix, which describes the mechanical coupling of individual atoms in the molecular junction at the microscopic scale, plays a key role. To obtain the dynamical matrix, we calculate the second derivative of the Born-Oppenheimer energy landscap $\mathrm{e}^{38,39}$ by using density functional perturbation theory, as implemented in the quantum chemistry software package TURBOMOLE, version $7.1^{40}$. Total energies are converged up to a precision of $10^{-9}$ a.u., and geometries are optimized until the change of the maximum norm of the Cartesian gradient is smaller than $10^{-5}$ a.u. We use the Perdew-Burke-Ernzerhof exchange-correlation functional ${ }^{41,42}$ and the default 2 basis set of split-valence-plus-polarization quality def2-SV $(\mathrm{P})^{43,44}$ in combination with the corresponding Coulomb fitting basis.

Junction geometries and pulling curves. All junction geometries studied in Fig. 4 are built up from two gold pyramids oriented in the crystallographic (111) direction: one end of the straight alkane chain is attached via a sulphur group to the tip atom of one of the pyramids, and the other end of the chain is attached via a sulphur atom to the other pyramid. The atomically sharp pyramids model probe and substrate metal electrodes close to the point of rupture, when they have been deformed by mechanical stress and gold atoms have been pulled out of the soft but initially flat substrate surface. The positions of the atoms in the central junction part, consisting of the molecule and the two metal layers closest to it, are optimized by energy minimization, while the $\mathrm{Au}$ atoms in the two outermost rows of the $\mathrm{Au}_{20}$ pyramids on each side, that is, those most distant from the molecule, are kept fixed. To generate the pulling curves of Fig. 4, the junctions are adiabatically stretched by displacing the frozen part of the gold atoms on one side of the molecular junction in the direction of the difference vector between the Au tip atoms with a step size of $d=0.5$ a.u. $\approx 0.26 \AA$, and optimizing again all the atoms in the central junction part under the new boundary condition set by the fixed outermost Au layers. We note that in these simulations, we mimic the experiments and obtain contact geometries that correspond to those manifested in single-molecule junctions at breakdown ${ }^{45}$.

Additional thermal conductance-distance traces. In Fig. 4 we present thermal conductance versus distance curves for molecular junctions of the five different molecules $\mathrm{C} 2-\mathrm{C} 10$. In each case a gold atom is pulled out from the Au electrodes to yield a short gold chain in the form of a dimer before the contact breaks. To inspect the robustness of the results, we performed further simulations of junction stretching processes and show in Extended Data Fig. 5 additional pulling curves for C2, $\mathrm{C} 6$ and $\mathrm{C} 10$. Here the gold atoms at the tips move from a three-fold hollow to a two-fold bridge position before the contact breaks, as is visible from the geometries displayed in the insets of Extended Data Fig. 5. We note that in this analysis the initial geometries differ from those shown in the main text with respect to the orientation of the molecule on the pyramidal leads, but the stretching protocol is otherwise identical. The computed thermal conductances at rupture are slightly higher than those shown in Fig. 4, since the blue region is missing, but they are 
within the uncertainty of the measured thermal conductance values (Fig. 3c). As these examples show, the formation of gold chains in our simulations depends sensitively on the starting geometry and the details of the adiabatic stretching process. A faithful reproduction of the mechanical deformation of the macroscopically large gold electrodes will require a larger number of flexible gold atoms than what we can at present use in our computationally demanding ab initio simulations.

Influence of variations of the contact geometry. As for any mechanically controlled break-junction technique, junction geometries in the experiment are not well controlled at the atomic scale, and the space of possible configurations is huge. This leads to uncertainties with regard to molecular configuration, moleculeelectrode coupling and electrode orientation. Assuming alkane molecules to be fully stretched before contact rupture, it is interesting to explore the variation of phonon thermal conductance as the geometry of the contacts is varied. In Extended Data Fig. 6 we show the computed changes in the energy-dependent phonon transmission for $\mathrm{Au}-\mathrm{C} 10-\mathrm{Au}$ single-molecule junctions with different contact geometries. Specifically, we find that peak positions and peak widths in the transmission spectrum depend on the precise atomic geometries.

In Extended Data Table 1, we present the computed thermal conductances for the four $\mathrm{Au}-\mathrm{C} 10-\mathrm{Au}$ junctions shown in Extended Data Fig. 6, but include also the data for corresponding junction types containing $\mathrm{C} 2-\mathrm{C} 8$. We also list the resulting standard deviations for each molecule that are found to be in the range of 3-7 $\mathrm{pW} \mathrm{K}^{-1}$ and in close correspondence to the standard deviation of the measured thermal conductances (see Fig. 3c). We note that in this analysis we designed the different junction types such that stress is minimized, in order to concentrate on the effects of metal-molecule binding and electrode orientation. The molecular contacts are therefore located inside the yellow-shaded area of Fig. 4, which results in a somewhat larger thermal conductance than those obtained in our experiments. Electronic contributions to the thermal conductance. In Fig. 4 of the main text we have estimated $G_{\text {thel }}$ with the help of the Wiedemann-Franz law, based on the mean experimental single-molecule electrical conductance value. The Wiedemann-Franz law reads $G_{\text {th,el }}=L_{0} T G_{\text {el }}$, where the Lorentz number $L_{0}=\pi^{2} k_{\mathrm{B}}^{2} / 3 e^{2}=2.44 \times 10^{-8} \mathrm{~W} \Omega \mathrm{K}^{-2}, T$ is the temperature, and $G_{\mathrm{el}}$ the electrical conductance. Using the experimental values $G_{\mathrm{el}}=10^{-2} G_{0}$ for $\mathrm{C} 2$, and $G_{\mathrm{el}}=2 \times 10^{-3} G_{0}$ for $\mathrm{C} 4$, in addition to $T=300 \mathrm{~K}$, we obtain the data for $G_{\text {th,el }}$ given in the text.

Transmission eigenchannels. To obtain further information about heat transport in nanosystems, we decomposed the phonon transmission function, $\tau_{\mathrm{ph}}(E)$, into energy-dependent contributions $\tau_{\mathrm{ph}, i}(E)$ of individual transmission eigenchannels $i$ :

$$
\tau_{\mathrm{ph}}(E)=\sum_{i} \tau_{\mathrm{ph}, i}(E)
$$

These eigenchannels are scattering states, and the transmission coefficients $0 \leq \tau_{\mathrm{ph}, i}(E) \leq 1$ are the eigenvalues of the transmission probability matrix ${ }^{29}$.

In Extended Data Fig. 7 we display, along with $\tau_{\mathrm{ph}, i}(E)$ for $i=1,2,3$, the most transmissive eigenchannel $i=1$ of C2, C6 and C10 at selected energies. These are the highest energies at which a transmission resonance occurs with a value close to 1 . Note that we show here a static representation of the eigenchannels in terms of the real part at time $t=0$, despite the general solution being complex or time-dependent. Similar to the discussion in our past work ${ }^{29}$, a close relation of the molecular vibrations to the transmission eigenchannels often exists. We observe that for $\mathrm{C} 2$ junctions only the centre-of-mass motions of the molecule contribute to phonon transport due to the short molecular length. However, for C6 and C10, genuine molecular modes carry heat. This is evident from direction changes of the arrows in Extended Data Fig. 7 that indicate the atomic motion, when going from one end of the molecule in the junction to the other.

\section{Code availability}

The DFT program used to analyse the electronic structure and vibrational properties is available from www.turbomole.com. The corresponding custom-developed code for the description of phonon transport implements the procedures outlined in ref. ${ }^{31}$ and is available from F.P. on reasonable request.

33. Cui, L. et al. Study of radiative heat transfer in Ångström- and nanometresized gaps. Nat. Commun. 8, 14479 (2017); correction 9, 16225 (2018).

34. Sadat, S., Meyhofer, E. \& Reddy, P. High resolution resistive thermometry for micro/nanoscale measurements. Rev. Sci. Instrum. 83, 084902 (2012).

35. Malen, J. A. et al. Identifying the length dependence of orbital alignment and contact coupling in molecular heterojunctions. Nano Lett. 9, 1164-1169 (2009).

36. Wang, J. S., Wang, J. \& Lü, J. T. Quantum thermal transport in nanostructures. Eur. Phys. J. B 62, 381-404 (2008).

37. Li, N. et al. Phononics: manipulating heat flow with electronic analogs and beyond. Rev. Mod. Phys. 84, 1045-1066 (2012).

38. Deglmann, P., Furche, F. \& Ahlrichs, R. An efficient implementation of second analytical derivatives for density functional methods. Chem. Phys. Lett. 362 , 511-518 (2002).

39. Deglmann, P., May, K., Furche, F. \& Ahlrichs, R. Nuclear second analytical derivative calculations using auxiliary basis set expansions. Chem. Phys. Lett. 384, 103-107 (2004).

40. TURBOMOLE version 7.1, http://www.turbomole.com (TURBOMOLE GmbH, Karlsruhe, 2016).

41. Perdew, J. P., Burke, K. \& Ernzerhof, M. Generalized gradient approximation made simple. Phys. Rev. Lett. 77, 3865-3868 (1996).

42. Perdew, J. P. \& Wang, Y. Accurate and simple analytic representation of the electron-gas correlation energy. Phys. Rev. B 45, 13244-13249 (1992).

43. Weigend, F. Accurate Coulomb-fitting basis sets for $\mathrm{H}$ to Rn. Phys. Chem. Chem Phys. 8, 1057-1065 (2006).

44. Weigend, F. \& Ahlrichs, R. Balanced basis sets of split valence, triple zeta valence and quadruple zeta valence quality for $\mathrm{H}$ to $\mathrm{Rn}$ : design and assessment of accuracy. Phys. Chem. Chem. Phys. 7, 3297-3305 (2005).

45. Paulsson, M., Krag, C., Frederiksen, T. \& Brandbyge, M. Conductance of alkanedithiol single-molecule junctions: a molecular dynamics study. Nano Lett. 9, 117-121 (2009).

Acknowledgements P.R. and E.M. acknowledge funding from the US Office of Naval Research (N00014-16-1-2672, instrumentation), the US Department of Energy (DE-SC0004871, scanning probe microscopy) and the US National Science Foundation (1803983). P.R. and E.M. acknowledge the Lurie Nanofabrication Facility and the Michigan Center for Materials Characterization for facilitating the fabrication and calibration of devices. S.-Y.J. gratefully acknowledges support from a National Research Foundation (NRF) grant funded by the Korean Government (no. 2016R1A5A1012966). J.C.K. and F.P. thank the Collaborative Research Center (SFB) 767 of the German Research Foundation (DFG) for financial support. A large part of the numerical modelling was carried out using the computational resources of the bwHPC programme, namely, the bwUniCluster and the JUSTUS HPC facility.

Author contributions The work was conceived by P.R. and E.M. The experiments were performed by L.C. The devices were fabricated by S.H. and W.J. The monolayer samples were prepared by Z.A.A. under the guidance of S.-Y.J. The calculations were performed by J.C.K. under the guidance of F.P. The manuscript was written by L.C., F.P., P.R. and E.M. with comments and inputs from all authors. 


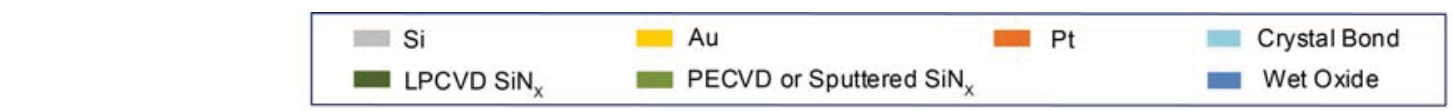
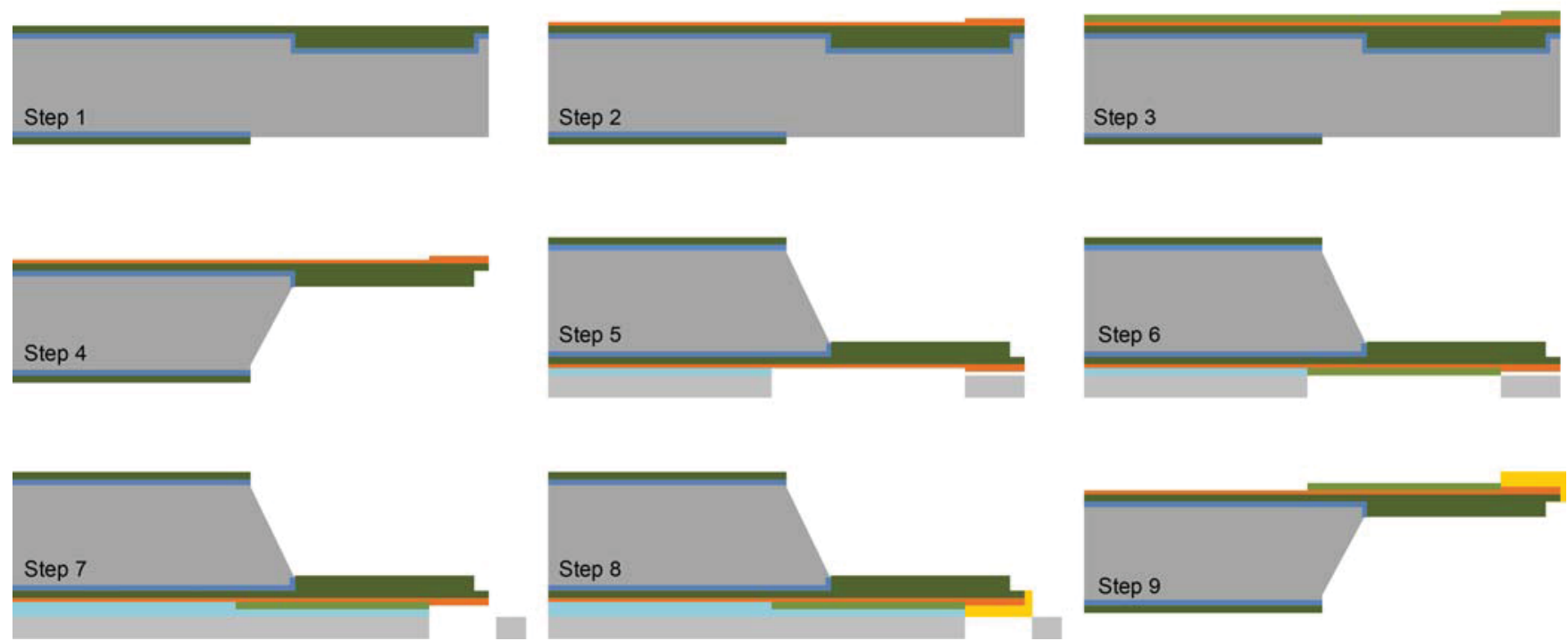

Extended Data Fig. 1 | Fabrication steps for thermal probes. Step 1, ' $\mathrm{T}$ '-shaped cantilever patterning. Step 2, deposition of Pt for the serpentine heater-thermometer, pads and the tip. Step 3, $\mathrm{SiN}_{x}$ layer deposition for front side $\mathrm{KOH}$ etching. Step 4, probe cantilever release. Step 5, aligning each probe on the first shadow mask using a thin low-temperature

crystal bond layer. Step 6, $\mathrm{SiN}_{x}$ sputtering on the serpentine Pt heaterthermometer. Step 7, aligning each probe on the second shadow mask. Step 8, Au sputtering on the tip region. Step 9, detaching the scanning probe from the shadow mask and removing the residual crystal bond by 'piranha' cleaning. 
a

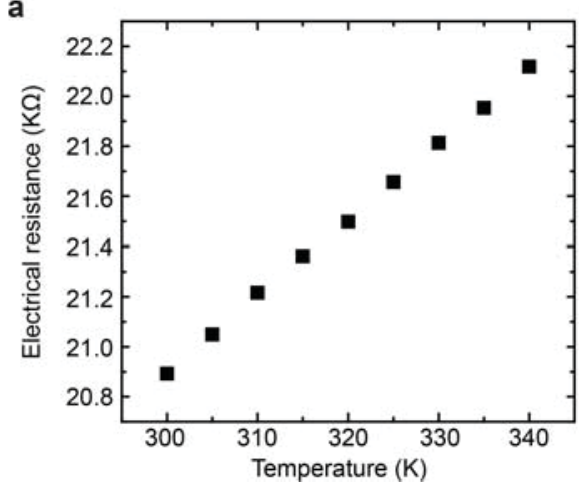

b

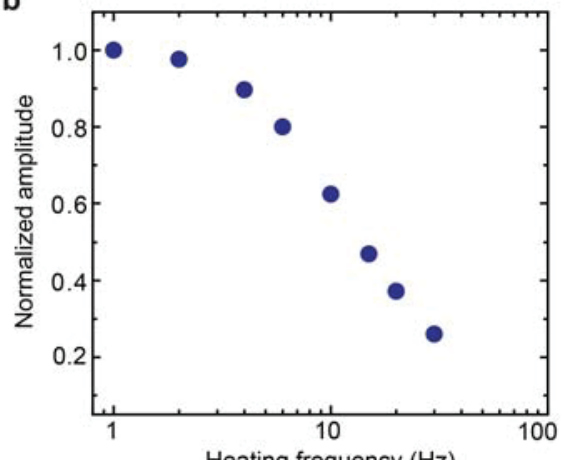

c

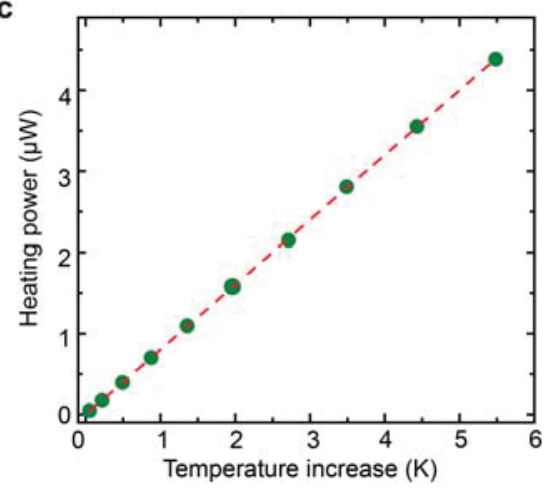

Extended Data Fig. 2 | Characterization of thermal and electrical properties of the scanning thermal probes. a, Measured electrical resistance of the Pt heater-thermometer as a function of temperature. b, Measured thermal response of the scanning probe as a function of the

heating frequency. c, Calibration of the thermal conductance of the probe (input heating power provided to the Pt heater-thermometer plotted against the temperature rise of the probe). The slope of the dashed fitted line corresponds to the thermal conductance of the probe. 


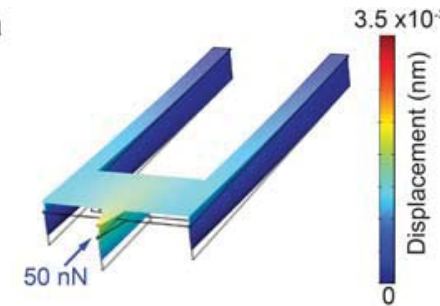

C

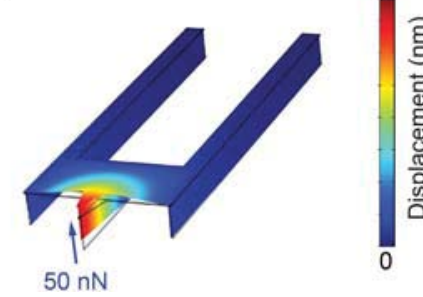

b

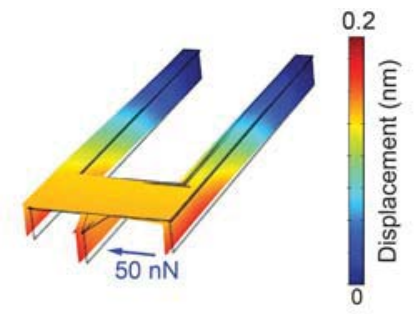

d

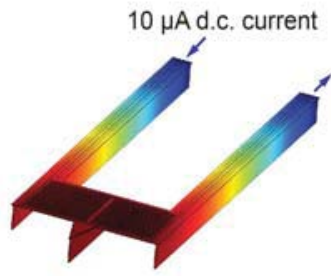

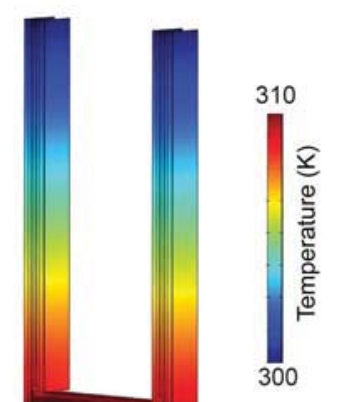

300
Extended Data Fig. 3 | Evaluation of mechanical properties and spatial temperature variation of the scanning thermal probes. a-c, A force of $50 \mathrm{nN}$ was applied either in the normal or the transverse directions of the beams, and the deflection for each case was computed ('Displacement', colour key). The stiffness of the probe was calculated to be $14,000 \mathrm{~N} \mathrm{~m}^{-1}$ (a), $275 \mathrm{~N} \mathrm{~m}^{-1}$ (b) and $12.5 \mathrm{~N} \mathrm{~m}^{-1}$ (c), respectively, for the normal and two transverse directions. d, e, Calculated temperature field (colour key at right) of the scanning thermal probe when a $10-\mu \mathrm{A}$ d.c. current was applied to the embedded serpentine Pt heater-thermometer (d) and a $10-\mu \mathrm{W}$ heat current was input from the tip (e). The spatial temperature distribution on the island is very uniform ( $<5 \%$ change across locations), supporting the expectation that the distributed Pt heater-thermometer accurately measures the temperature of the suspended region. 


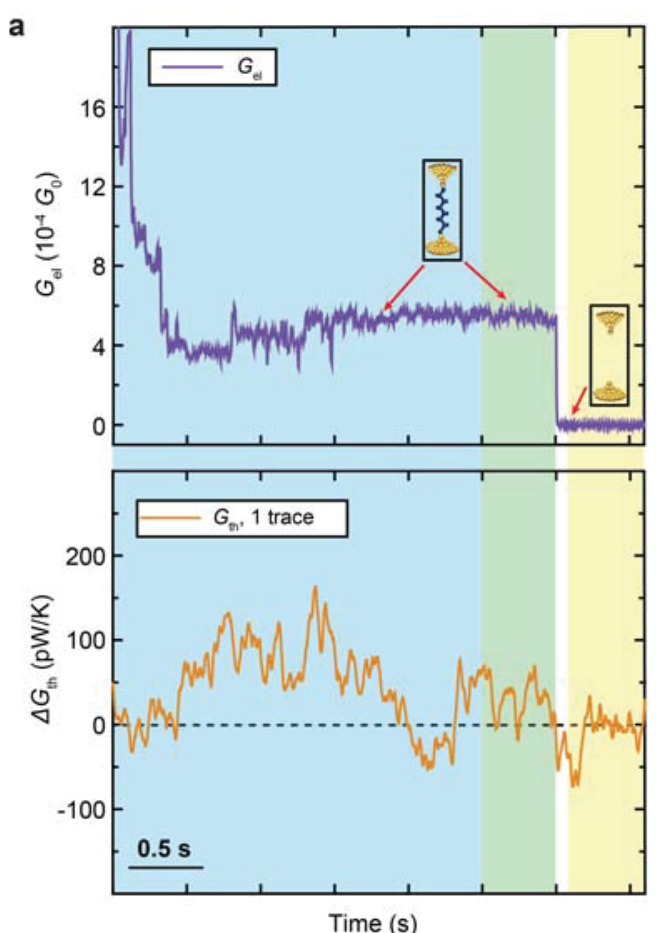

Extended Data Fig. $4 \mid$ Sample electrical and thermal conductance traces for Au-C6-Au molecular junctions. a, b, Two independent sample recordings. Top and bottom panels show electrical and thermal conductance, respectively. The green- and yellow-shaded regions mark portions of the recordings that capture the rupture of a single-molecule junction to which the time-averaging scheme is applied, while the blue-shaded regions during the earlier portions of the withdrawal cycle represent recordings that contain events involving multi-molecule
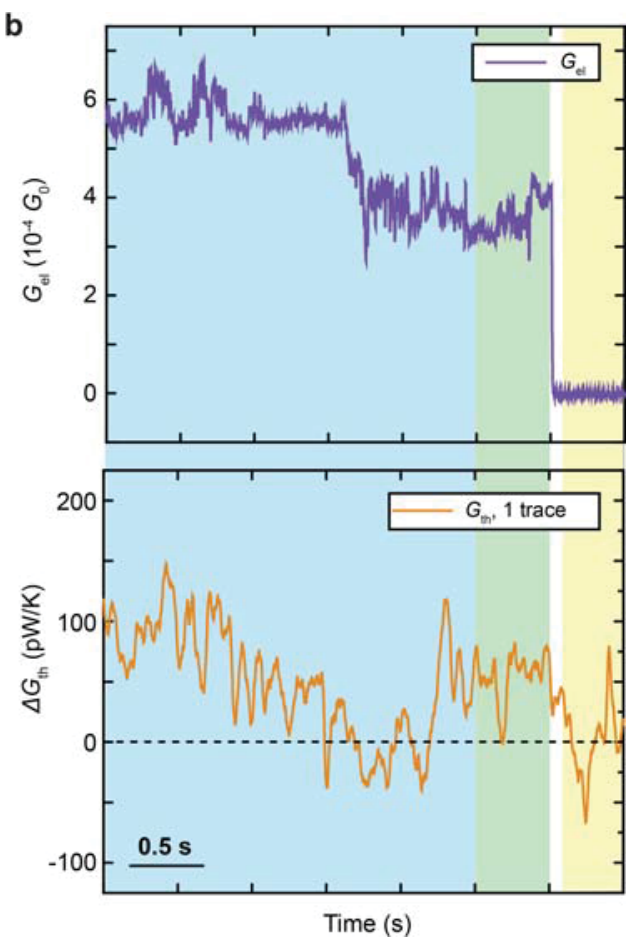

junctions. A clear last step can be identified in the electrical conductance traces (green-yellow region), indicating the breakdown of a singlemolecule junction. As can be seen, there are also additional steps before the last step in the blue-shaded region. The corresponding thermal conductance traces that are shown below each electrical conductance trace do not reveal any thermal conductance steps, owing to the low signal-tonoise ratio. Insets, schematics of single-molecule junctions before and after rupture. 


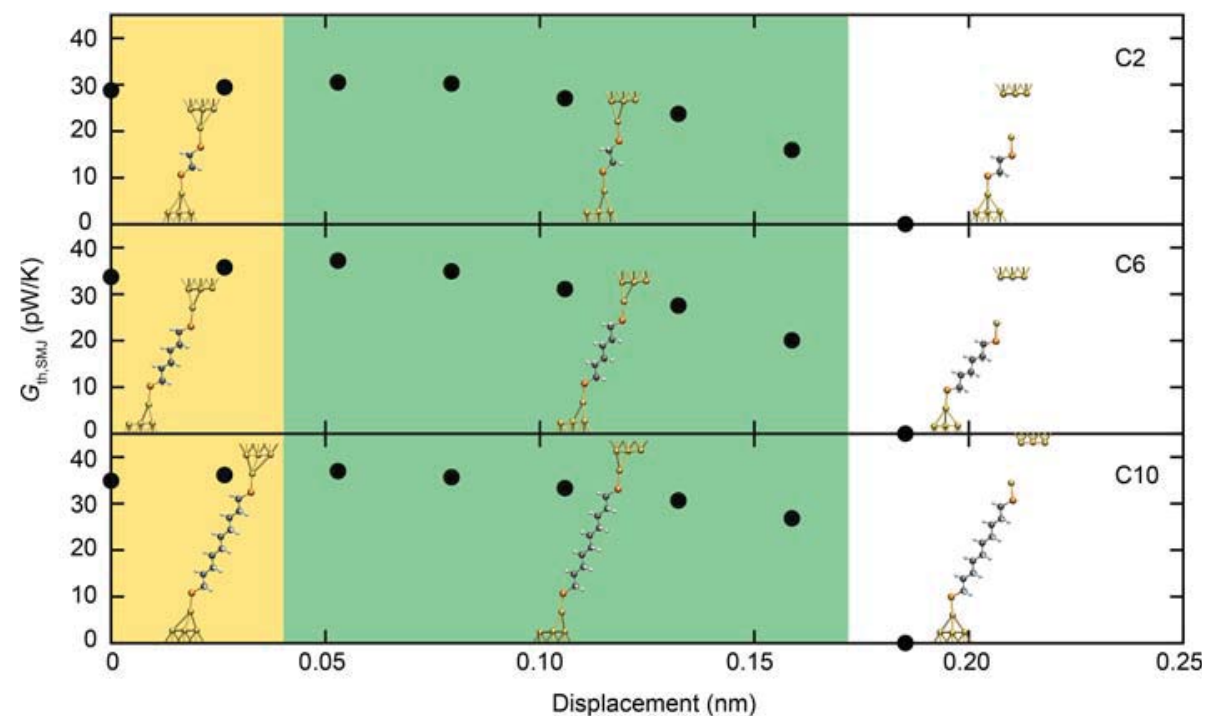

Extended Data Fig. 5 | Simulated thermal conductance as a function of electrode displacement for $\mathrm{C} 2, \mathrm{C} 6$ and $\mathrm{C} 10$ single-molecule junctions.

The computed thermal conductance data are shown as black dots,

junction structures are displayed as insets. The initial junction geometries before displacement of the electrodes differ from those used to generate coloured regions have the same meaning as in Fig. 4, and snapshots of the corresponding plots in Fig. 4, but the procedure employed for stretching the junctions is the same. 
a

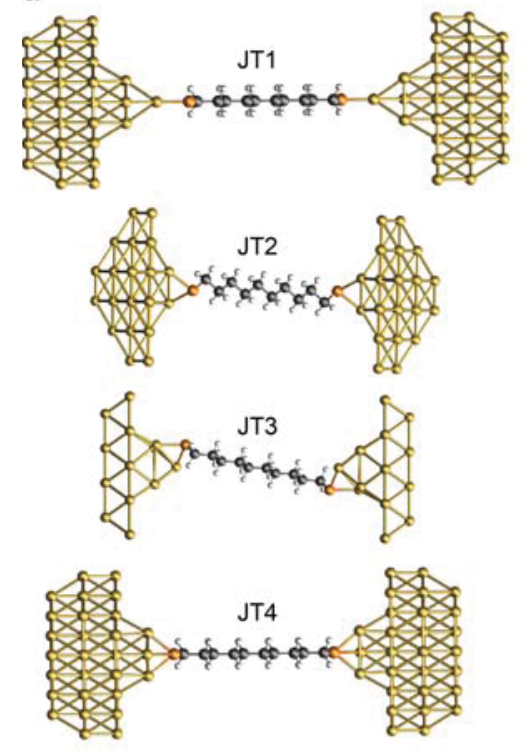

b

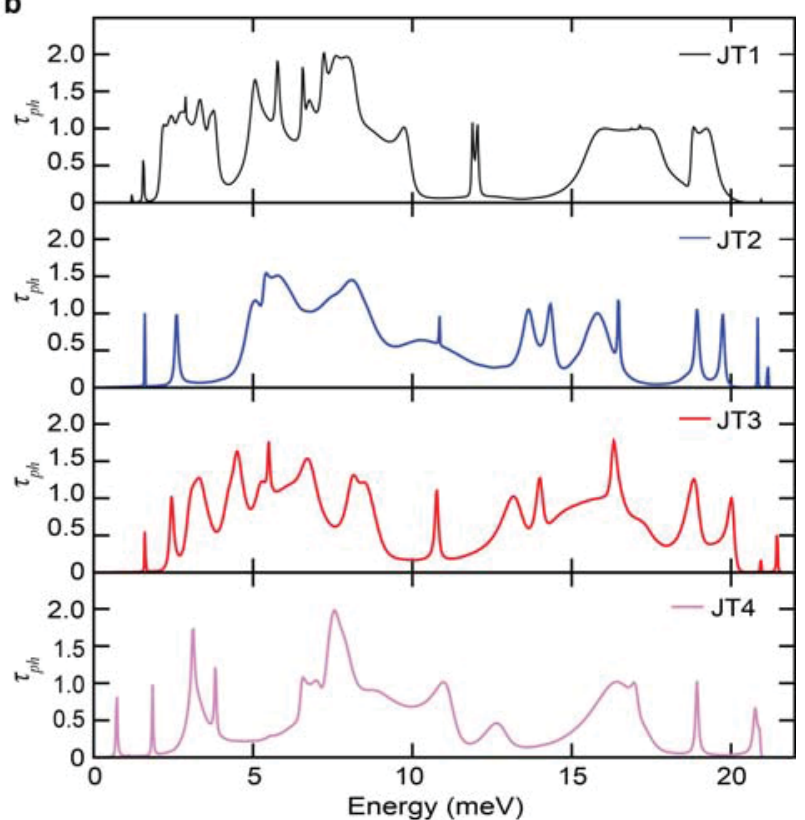

Extended Data Fig. 6 Influence of the contact geometry on computed phonon transmission for $\mathrm{Au}-\mathrm{C} 10-\mathrm{Au}$ single-molecule junctions.

a, Different junction types that are used to evaluate the effect of contact geometry on the phonon transmission functions. Each terminal sulphur atom in the junction is attached to a single Au tip atom (JT1), to two Au tip atoms (JT2, JT3) or to three Au atoms (JT4). In these geometries, electrodes are oriented along the (111) crystallographic direction (JT1, JT4), the (110) direction (JT2) and the (100) direction (JT3). b, Phonon transmission as a function of energy for the different junction geometries illustrated in $\mathbf{a}$. 


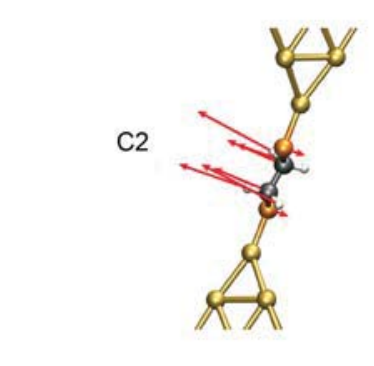

b

d

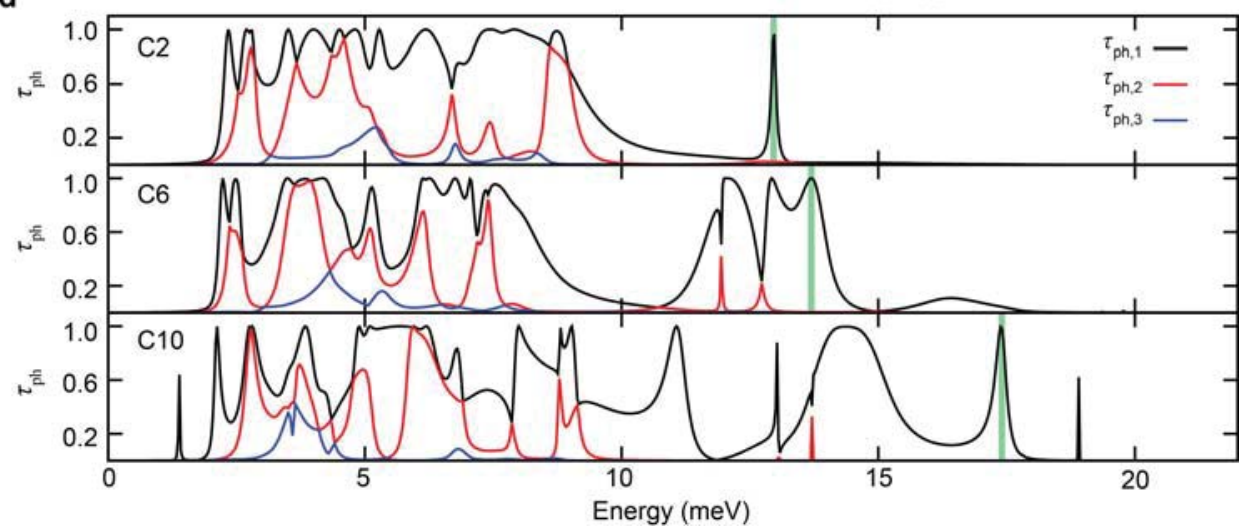

c

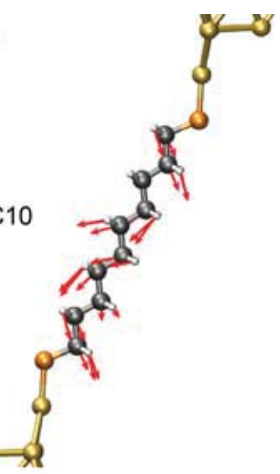

C6

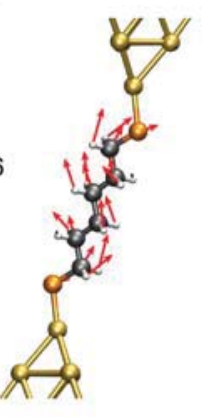

\section{,}

Extended Data Fig. 7 | Phonon transmission eigenchannels for C2, C6 and C10 junctions. a-c, Displacement patterns associated with the mode shapes of the most transmissive eigenchannel $i=1$ for $\mathrm{C} 2$, C6 and C10, respectively, evaluated at energies of $13.5 \mathrm{meV}, 14 \mathrm{meV}$ and $18 \mathrm{meV}$. d, Phonon transmission associated with each of the three eigenchannels $i=1,2,3$ for C2, C6 and C10 molecular junctions. A peak in th

in the transmission of eigenchannel $i=1$ is found to occur at energies of around $13.5 \mathrm{meV}, 14 \mathrm{meV}$ and $18 \mathrm{meV}$ for C2, C6 and C10 junctions, respectively, and is indicated by the green bars. The displacement patterns of transmission eigenchannel $i=1$ in a-c have been evaluated at these energies. 
Extended Data Table 1 | Calculated thermal conductance

\begin{tabular}{|c|c|c|c|c|c|}
\hline Molecule & $\begin{array}{r}G_{\text {th, JT1 }} \\
(\mathrm{pW} / \mathrm{K})\end{array}$ & $\begin{array}{l}G_{\text {th }, \pi 2} \\
(p W / K)\end{array}$ & $\begin{array}{l}G_{\text {th, JT3 }} \\
(\mathrm{pW} / \mathrm{K})\end{array}$ & $\begin{array}{l}G_{\text {th }, J T 4} \\
(p W / K)\end{array}$ & $\begin{array}{c}\text { Standard } \\
\text { Deviation } \\
(\mathrm{pW} / \mathrm{K})\end{array}$ \\
\hline $\mathrm{C} 2$ & 28.0 & 21.9 & 37.7 & 30.4 & 6.5 \\
\hline $\mathrm{C} 4$ & 29.7 & 30.9 & 41.4 & 41.0 & 6.3 \\
\hline C6 & 33.9 & 31.7 & 36.9 & 40.3 & 3.7 \\
\hline $\mathrm{C} 8$ & 35.5 & 35.5 & 42.2 & 32.0 & 4.2 \\
\hline C10 & 44.0 & 34.8 & 45.3 & 32.7 & 6.4 \\
\hline
\end{tabular}

Columns 2-5 show the calculated thermal conductance of Au-alkanedithiol-Au single-molecule junctions for four types of junction geometries, respectively JT1, JT2, JT3 and JT4. The entry in column 1 shows the alkanedithiol molecule concerned. Junction geometries are either identica to those shown in Extended Data Fig. 6 (for C10) or similar to them (C2-C8). Each terminal sulphur atom attaches to a single Au tip atom for JT1, to two Au tip atoms for JT2 and JT3, or

to three Au tip atoms for JT4, while the electrodes are oriented along the (111) crystallographic direction for JT1 and JT4, the (110) direction for JT2 or the (100) direction for JT3. The standard deviation (column 6 ) indicates the variability of the thermal conductance as determined for each molecule from the four different junctions types. 